\title{
MICRO-RADAR AND UWB AIDED UAV NAVIGATION IN GNSS DENIED ENVIRONMENT
}

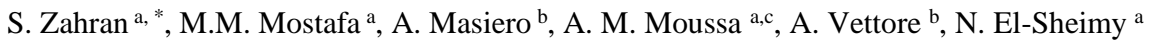 \\ ${ }^{a}$ Department of Geomatics, University of Calgary, Calgary, Canada - (shady.zahran1, mostafa.mostafa, elsheimy)@ucalgary.ca \\ b Interdepartmental Research Center of Geomatics (CIRGEO), University of Padova, \\ Viale dell’Università 16, Legnaro (PD) 35020, Italy - (masiero@ dei.unipd.it , antonio.vettore@unipd.it) \\ ${ }^{\mathrm{c}}$ Department of Electrical Engineering, Port-Said University, Port Said, Egypt - amelsaye @ ucalgary.ca
}

\section{Commission I, WG I/6}

KEY WORDS: Micro-Radar, Ultra-Wideband, Multi-Sensor Fusion, Extended Kalman Filter, Global Navigation Satellite System, Inertial Measurement Unit.

\begin{abstract}
:
During the last decade, the number of applications of UAVs has continuously increased, making the global UAV market one of those with the highest rate of growth. The worldwide increasing usage of UAVs is causing an ever-growing demand for efficient solutions in order to make them usable in every kind of working condition. In fact, nowadays the main restriction to the usage of UAVs is probably the need of reliable position estimates provided by using the Global Navigation Satellite System (GNSS): since UAVs mostly rely on the integration of GNSS/Inertial Navigation System (INS) to properly fulfil their tasks, they face a major challenge while navigating in GNSS denied environments. The goal of this paper is that of investigating possible strategies to reduce such main restriction to UAV usage, i.e. enabling flights in GNSS denied environment by providing position estimates with accuracy quite comparable to that of standard GNSS receivers currently mounted on commercialized drones. To be more specific, this paper proposes the combined use of a novel Frequency Modulated Continuous Wave (FMCW) Radar, a set of Ultra-Wideband (UWB) devices, and Inertial Measurement Unit (IMU) measurements in order to compensate for the unavailability of the GNSS signal units. A 24-GHz micro FMCW radar and a UWB device have been attached to a quadcopter during the flight. The radar receives the reflections from ground scatterers, whereas the UWB system provides range measurements between a UWB rover mounted on the UAV and a set of UWB anchors distributed along the flying area.
\end{abstract}

\section{INTRODUCTION}

During the past decade, there has been an enormous increase in the applications utilizing fully autonomous Unmanned Aerial Vehicles (UAVs). Despite initially these applications were mostly restricted to military fields like border surveillance, troops enforcement, combat, target, and decoy, they have been quite recently extended to many civilian applications, such as firefighting, traffic monitoring, and commercial UAVs. This wide variety of applications makes the UAVs autonomous navigation a challenging task, as the UAVs mainly depends on the integration between GNSS and IMU to navigate autonomously, but during the lack or unavailability of the GNSS signals, the UAVs lose its ability to navigate autonomously, due to the massive drift exhibited by the IMU when working in dead reckoning mode. GNSS signals may be unavailable due to jamming, spoofing, blockage, or multipath. So, another aiding system must be integrated with the IMU, to yield a reliable navigation solution that makes the UAV capable of fulfilling its assigned tasks in a wider range of working conditions.

Different sensors and methods were utilized to aid the navigation solution during GNSS outage, Light Detection and Ranging sensor (LIDAR) is one of the main utilized sensors to be integrated with IMU during these periods (Kumar, Patil, Patil, Park, \& Chai, 2017). The main drawback facing the use of LIDAR with small and micro UAVs, is the weight, size, power consumption, and high computational cost. A common approach that utilizes LIDARs to aid the vehicle to navigate is Simultaneous Localization and Mapping (SLAM) (Mohamed, Moussa, Elhabiby, El-Sheimy, \& Sesay, 2016) (Gao, Liu, Atia, $\&$ Noureldin, 2015), SLAM is a method to determine the vehicle navigation state, and constructing a map for the surrounding simultaneously (Cadena et al., 2016).

Camera is also considered as a viable solution to aid in navigation during the GNSS outage periods, as it is characterized by features that makes it suitable for small and micro UAVs like light weight, small size, and low power consumption, also it provides rich information like features and colors. Visual information provided by a camera can be used for 3D surrounding construction, obstacle avoidance, and position estimation. Position estimation can be classified into two groups; Vision Aided Navigation (VAN) and Vision-Based Navigation (VBN), VBN mainly depends on the camera only while VAN depends on the integration between camera and other sensors like IMU (Mostafa, Moussa, El-Sheimy, \& Sesay, 2017). One of the algorithms mostly used for exploiting camera in UAV navigation is Visual Odometry (VO), which depends on the extraction of the vehicle velocity from matching features of successive images (Aqel, Marhaban, Saripan, \& Ismail, 2016) (Nistér, Naroditsky, \& Bergen, 2004). One of the biggest problems facing using a single camera is the loss of scale information: despite this issue can usually be resolved by using stereo/multiple cameras (Schauwecker \& Zell, 2014), this solution is not so effective for small and micro UAVs, because its accuracy mainly depends on the base distance between the cameras, which is necessarily limited in such cases. Regardless of using single/multiple cameras, this sensor performance is still greatly sensitive to certain environmental factors, like light variation or featureless scenes.

Other sensor which is much more superior to cameras or LIDAR, because it is more immune to environmental changes is Radar (Barrett \& Alvarez, 2017). In the past Radar was not used with small/micro UAVs due to its weight, size, and power

${ }^{*}$ Corresponding author. 
consumption, which usually makes it not suitable for this kind of platform. However, very recent radar technological developments enabled the manufacture of small/micro radars, that are suitable to be mounted on small/micro UAVs and, consequently, to be usable for bounding the drift of the IMU solution. However, there are a lot of challenges to utilize such sensor in navigation, like the huge amount of data, the existence of large amount of clutters especially when flying at low altitude, accuracy of the information obtained from radar, and how to integrate this information to aid in navigation.

In (Fasano et al. 2017), to prove the ability of micro radar to yield useful information that may be utilized in navigation, detection of objects, surveillance, or obstacle avoidance, they mount a micro radar (PulsON 440) on a small hex-copter UAV, and they showed the ability to use this radar to localize, identify and detect 3 metal objects placed on the ground from the radar imagery scene. Instead, (Scannapieco et al. 2016) used a small quadcopter equipped with micro frequency modulated continuous wave (FMCW) radar. The radar information is composed of ranges and angles of the detected targets. To remove the clutters, they used the constant false alarm rate (CFAR) filter applied on a sliding window (size of sliding window changes according to application): within this sliding window, a target is detected only if the power of the received signal exceeds a certain threshold, otherwise it is considered a false alarm. After this step a multiple target tracking (MTT) algorithm is applied, followed by signal value decomposition (SVD) to get the translation and rotation occurred, that indicate also the vehicle translation and rotation. This information is then fused with IMU through the extended Kalman filter (EKF) to achieve a better navigation performance during GNSS signal blockage. During their first experiments, they placed the radar looking forward, to decrease the amount of clutter, while in the second one they tilted the radar by 20 degrees, these configurations impose some limitations on the UAV, as it must either locate targets in front of the UAV or to fly near the ground to receive echoes when utilizing the second configuration. In addition to these limitations, when the UAV performs small movement, the difference between translation and rotation cannot be accurately estimated.

While in (Kauffman et al. 2013), to localize the vehicle, they developed a SAR based algorithm to process the simulated data from two side looking ultra-wideband radars, then these data were used to enhance the vehicle navigation.

Despite being initially used mostly for indoor terrestrial navigation, UWB devices have been recently considered in several UAV positioning works. Several works considered the use of UWB devices for improving UAV autonomous landing ability: (Li et al., 2015, Shin et al, 2017) used UWB radar-based solutions for such aim. Similarly, (Kim and Choi, 2016) used UWB time-of-flight measurements to improve the auto-landing of unmanned aircraft. (Benini et al, 2013) integrated UWB with IMU and visual odometry for mini-UAVs indoor localization. Despite the integration of such sensors is very interesting, the system has been used only in a small area (less than $100 \mathrm{~m}^{2}$ ), and visual odometry has been implemented by using small QR code markers, which, due to possible illumination issues and to the distance from the ground, is probably a not so convenient solution for outdoor applications. (Tiemann et al., 2015) also used UWB devices for indoor navigation of UAVs, however given the usage of only UWB, the usage of such system is limited to small areas (less than $100 \mathrm{~m}^{2}$ in their experiment). (Goel et al., 2016) presented a cooperating positioning system where UAVs exploit INS, WiFi and UWB radio measurements are used obtaining a $5 \mathrm{~m}$ positioning accuracy (in this case accuracy is probably limited by the low number of considered UWB devices). Several works considered also UWB devices for controlling flight formation of groups of UAVs and for determining their relative distances (Gross et al., 2015).

\section{SYSTEM OVERVIEW}

This work combines the use of radar (as radar odometry) and UWB systems (CUPT), as both systems complement each other. While the odometer updates provided by the radar can limit the INS drift to some extent, it still suffers from orientation drift if not updated regularly with position or heading updates.

Differently, UWB systems have quite strict infrastructure requirements to be effective as standalone positioning systems: a large number of beacons have to be displaced along all the vehicle trajectory to ensure a suitable number of beacons in view to act as CUPT update for the UAV, making such infrastructure requirements quite expensive and difficult to be satisfied in several conditions of interest.

Taking into account the above observations, in this work a combination of radar and UWB is proposed in order to aid each other when there is less number of beacons in view.

\subsection{Radar Data Acquisition}

The radar outputs range doppler map (RDM), that represents ranges and velocities. These measurements are calculated by transmitting a frequency modulated sawtooth signal, the time delay between the transmitted and the received signal represents the ranges as shown in equation (1), and the Doppler frequency (change in frequency between the transmitted and received signal) is used to calculate the velocity as shown in equation (2).

$$
R=\frac{c_{0} \Delta t}{2}
$$

Where:

$\mathrm{c}_{0}=$ speed of light $=3 \cdot 10^{8} \mathrm{~m} / \mathrm{s}, \Delta \mathrm{t}=$ measured time difference between transmitted and received signal [s] $\mathrm{R}=$ slant range antenna - aim $[\mathrm{m}]$

$$
\triangle f=\frac{\triangle v}{c} f_{0}
$$

Where:

$\triangle f$ is the change in frequency between the transmitted and the received signal, $\Delta v$ is the change in velocity between the transmitted and received signal, $f_{0}$ is the frequency of the emitted signal, $\mathrm{c}$ is the speed of the signal in the propagation medium.

The velocity is calculated based on image processing of the RDM where it is binarized based on a threshold (experimental) for targets detection purpose and clutter removal. The radial velocities for these detected targets are then obtained directly from the horizontal axis of the range Doppler map as shown in Figure 1. The vehicle forward velocity is estimated by projecting these radial velocities toward the forward direction by the radar tilting angle (60 degrees) in addition to the UAV pitch angle then averaging them to obtain the resultant component.

\subsection{UWB System}

The UWB positioning system used in this paper is composed of six Pozyx anchors and a rover mounted on the UAV (Pozyx Labs). It is worth to notice that, most of the UWB-based UAV positioning systems previously considered in the literature use quite expensive UWB devices (typically more than 1000 \$ per device), whereas Pozyx devices are much cheaper (approximately $150 \$$ per device). 

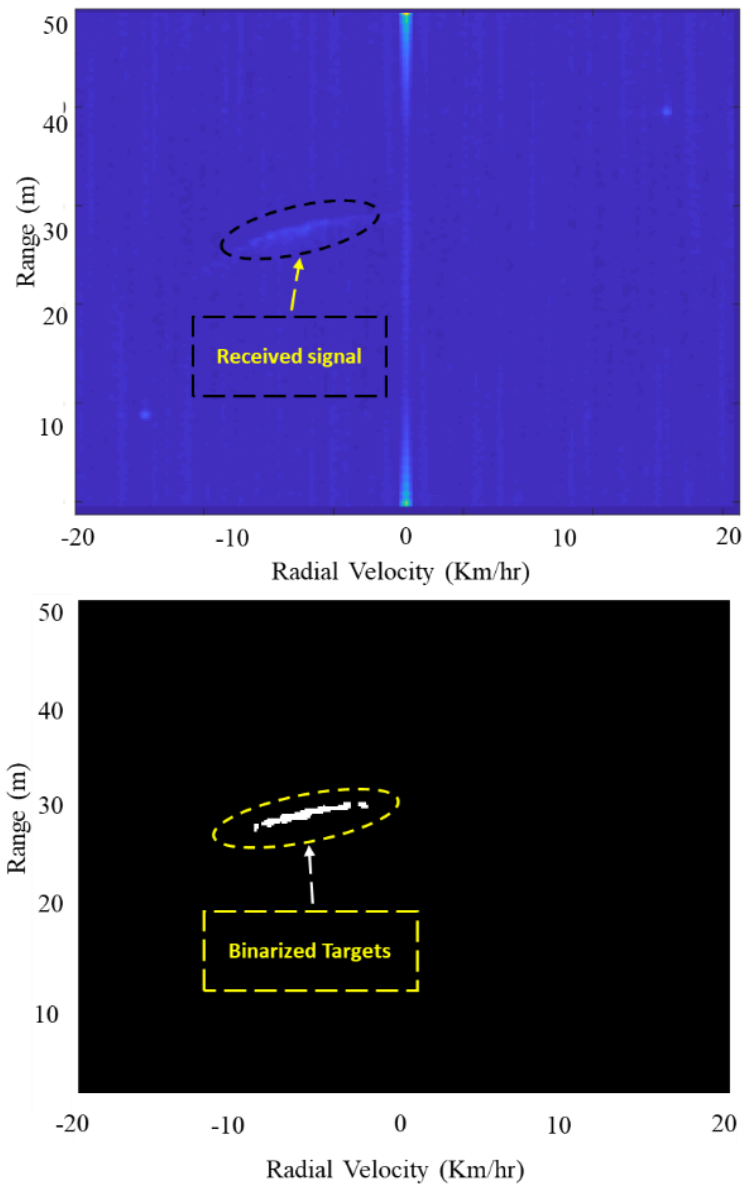

Figure 1: Range doppler map obtained from radar, and target binarization to calculate forward velocities of the vehicle.

Despite their performance is probably a bit worse than higher grade devices, their relatively low cost eases the development of much larger UWB networks.

Each UWB Pozyx device is a small and lightweight radio transmitter-receiver: assuming the radio propagation media (e.g. air) to be known and homogenous, and the two devices to be in clear-line-of-flight conditions (i.e. the line directly connecting such two devices is not obstructed by any obstacle), then the distance between such devices can be measured by means of the time-of-flight of a radio signal communication between them.

Then, assuming anchor positions to be constant and known, range measurements between each anchor and the rover can be used in order to estimate rover position by solving a standard trilateration problem.

Since UAVs usually have relatively smooth trajectories, the usage of an appropriate dynamic model inside of an EKF can often significantly improve the performance of UWB-based position estimates (Masiero et al, 2017).

Given the low-power transmission of UWB devices, their usage has been mostly focused to indoor environments, where distances between devices are relatively small, but the clear-line-of-sight working condition is often not satisfied because of the presence of several objects (and moving persons). Interestingly, such a working condition is usually easier to be guaranteed in UAV applications, hence potentially reducing outlier issues in UWB measurements.

\subsection{Data Fusion}

The information obtained from the radar (vehicle forward velocity), and the information form the UWB beacons (position of the vehicle) are fused with the output of the mechanization process through EKF.

The EKF is composed of 21 states (error states) as shown.

$$
\delta x=\left[\delta \mathrm{P}^{\mathrm{n}} \delta \mathrm{V}^{\mathrm{n}} \delta \varepsilon^{\mathrm{n}} a_{b} g_{b} a_{s} g_{s}\right]^{\prime}
$$

Where:

$\delta \mathrm{P}^{\mathrm{n}}, \delta \mathrm{V}^{\mathrm{n}}, \delta \varepsilon^{\mathrm{n}}$ represent the position, velocity and attitudes error vector, $a_{b}$ and $g_{b}$ are the accelerometers and gyroscopes biases respectively, $a_{s}$ and $g_{s}$ are the scale factor of the accelerometer and gyros.

The prediction step in the EKF system is obtained by linearizing the mechanization equations to obtain the system model, using first-order differential equation as shown in equation (4), and its discretization form is shown in equation (5):

$$
\begin{gathered}
\dot{x}=F x+G w \\
\hat{x}_{k}^{-}=\Phi_{k, k-1} \hat{x}_{k-1}+G_{k-1} w_{k-1}
\end{gathered}
$$

Where:

$x$ is the error states, $\mathrm{F}$ and $\Phi$ is the dynamic and state transition matrices, $\mathrm{G}$ is the noise coefficient matrix, $w$ is the system noise. First order Gauss-Markov (GM) model is used to represent the biases and scale factor of the accelerometers and gyroscopes.

The prediction of the state covariance matrix can be calculated as follows:

$$
P_{k}^{-}=\Phi_{k, k-1} P_{k-1} \Phi_{k, k-1}^{T}+G_{k-1} Q_{k-1} G_{k-1}^{T}
$$

Where:

$P$ is the state covariance matrix, $Q$ is the system noise $(w)$ covariance matrix.

The prediction step is followed by an update step, when more information is available (as velocity from radar or position form UWB system) as shown in equations (7, 8, and 9).

$$
\begin{gathered}
K_{k}=P_{k}^{-} H_{k}^{\mathrm{T}}\left(H_{k} P_{k}^{-} H_{k}^{\mathrm{T}}+R_{k}\right)^{-1} \\
\hat{x}_{k}=\hat{x}_{k}^{-}+K_{k}\left(Z_{k}-H_{k} \hat{x}_{k}^{-}\right) \\
P_{k}=\left(I-K_{k} H_{k}\right) P_{k}^{-}
\end{gathered}
$$

Where:

$K_{k}$ is the Kalman gain, $R_{k}$ is the covariance matrix of the measurements, $H_{k}$ is the design matrix, $Z_{k}$ is the observation matrix

\subsection{Hardware Setup}

A micro- Frequency Modulated Continuous Wave Radar (FMCWR) is attached to the bottom of the UAV during the flights. The radar operates at $24-\mathrm{GHz}$ frequency with one transmitter and three receiving antennas. The radar field of view is +/- 10 degree in azimuth and +/- 15 degree in elevation. The radar is capable of measuring the distances up to $300 \mathrm{~m}$ and $100 \mathrm{~m}$ for vehicles and persons respectively. The main benefits of utilizing such small radar are that it has light weight, small size, low power consumption, and it is immune to the environmental changes such as rain, fog, and dust. In addition, the radar has the ability to work during both day and night.

On the other hand, the UWB system is used to provide range measurements between an UWB rover mounted on the UAV and a set of six UWB anchors distributed on the flying area. UWB anchor positions were measured by means of a Trimble R10 GNSS receiver (accuracy $8 \mathrm{~mm} \mathrm{H} / 15 \mathrm{~mm} \mathrm{~V}$ ), hence 3D rover 
position can also be directly computed by means of UWB measurements when at least four of them are available, by solving a standard trilateration problem. Thanks to the small size (less than $10 \mathrm{~cm}$ per side) and light weight (approximately $12 \mathrm{~g}$ ), the Pozyx devices are very well suited for being mounted on drones with quite limited payload. Range measurement acquisition frequency is approximately $50 \mathrm{~Hz}$ when devices are close to each other, however, the low signal to noise ratio (transmission power is quite low) causes the number of received range measurements to significantly decrease for larger device distances. In practice, such decrease of available measurements imposes restrictions on the maximum flight altitude (Masiero et al, 2017).

The flight tests have been performed with a commercial on shelf UAV (3DR SOLO Quadcopter), which is equipped with Pixhawk-2 autopilot based on a MEMS-IMU and a $5 \mathrm{~Hz}$ U-blox GPS for navigation. The useful payload during the flight includes the micro-radar system and the UWB system (Rover), both systems were mounted on the quadcopter belly as shown in Figure 2. The radar data are processed with the aid of the onboard PC (Odoo X86 Ultra) through an ethernet connection.

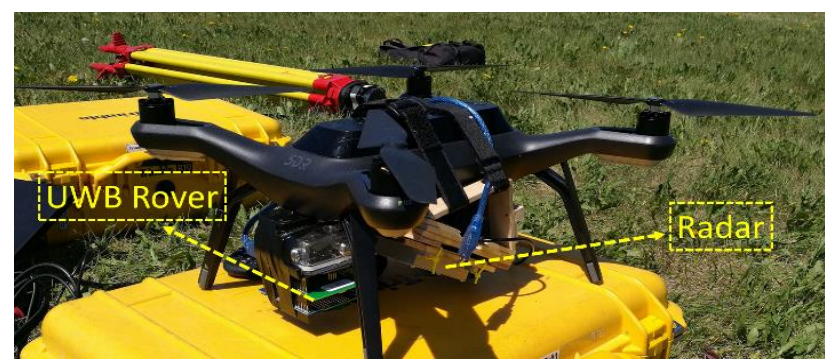

Figure: 3dr Solo quadcopter equipped with micro radar and UWB rover

\section{EXPERIMENTS AND RESULTS}

The experimental flight considered in this section and the UWB anchor distribution. Flight duration was $140 \mathrm{sec}$, with 19 waypoints. Three test scenarios have been considered in order to provide a comprehensive assessment of the system performance and characteristics. Figure 3 shows the estimated velocity obtained from the radar for the trajectory shown in Figure 4.

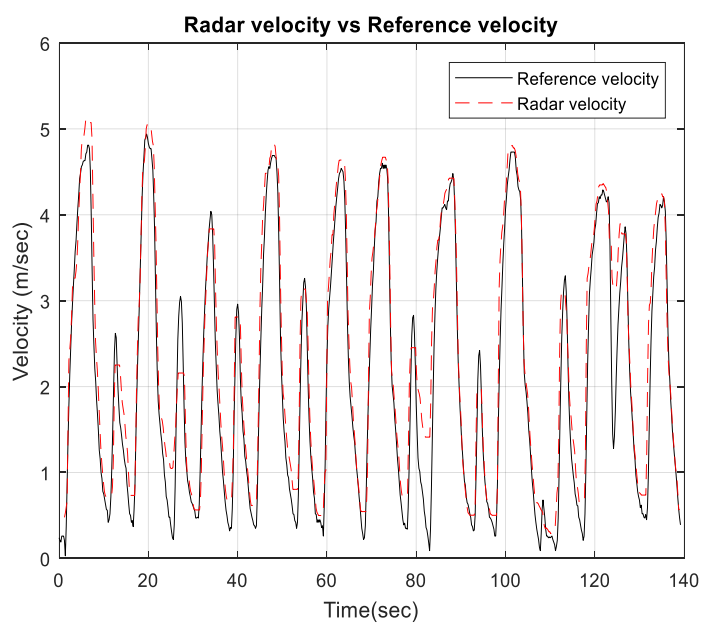

Figure 2 . Velocity estimated by radar vs Reference velocity

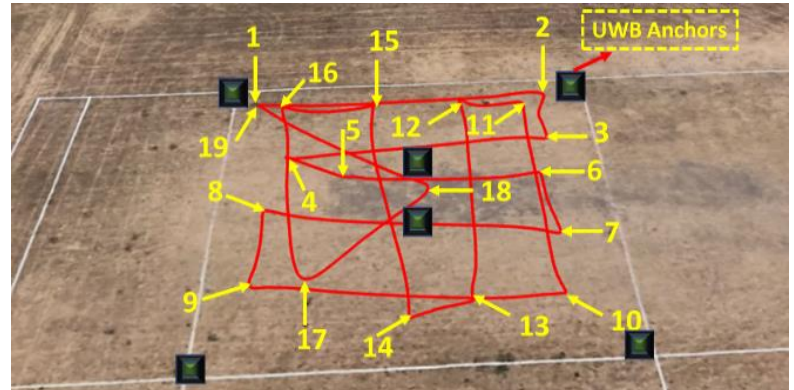

Figure 3. Flight trajectory

\subsection{First test scenario}

The first test scenario is to evaluate the performance of the IMU only when working as dead reckoning, during GNSS signal blockage.

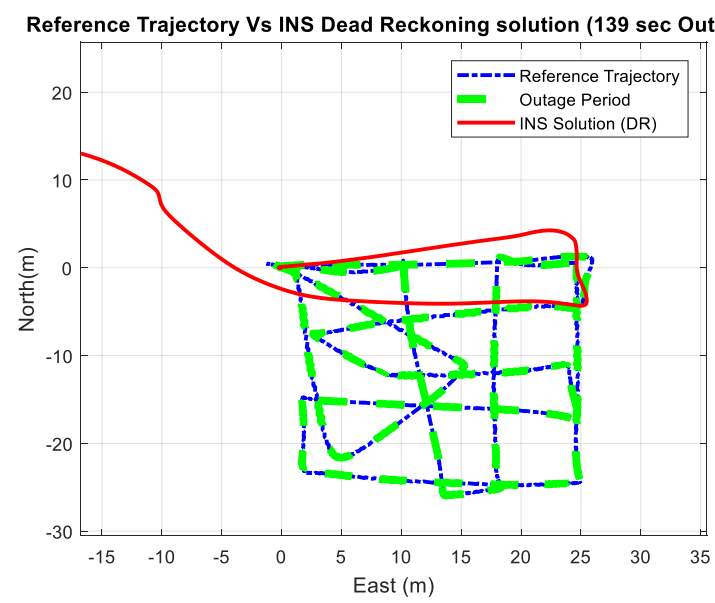

Figure 4. IMU dead reckoning solution

\begin{tabular}{|c|c|c|c|}
\hline RMSE-North & RMSE-East & $\begin{array}{c}\text { Max-Error- } \\
\text { North }\end{array}$ & $\begin{array}{c}\text { Max-Error- } \\
\text { East }\end{array}$ \\
\hline $1334.8 \mathrm{~m}$ & $1157.1 \mathrm{~m}$ & $3108.3 \mathrm{~m}$ & $3060.7 \mathrm{~m}$ \\
\hline
\end{tabular}

Table 1. IMU performance as dead reckoning

As seen in previous Figure 5 and Table 1, IMU solution as dead reckoning exhibited massive drift due to the accumulated errors, as the errors in north and east reached several thousands of meters. That's why the IMU needs an aiding system like GPS, Radar, LIDAR, or UWB to limit this error.

\subsection{Second test scenario}

In this test, only the radar is used as a velocity update system during the whole the flight trajectory, in order to aid the UAV to reach a better estimate of the navigation unknowns as shown in Figure 6 and 7, and table 2 .

This second test proves the great enhancement occurred in navigation when integrating the radar with INS, comparing it to the dead reckoning solution in the first test. As the errors in the north and east directions decreased from thousands (INS dead reckoning mode) to some meters (INS/radar integration). However, the whole trajectory is tilted, because the heading direction is not observed. 


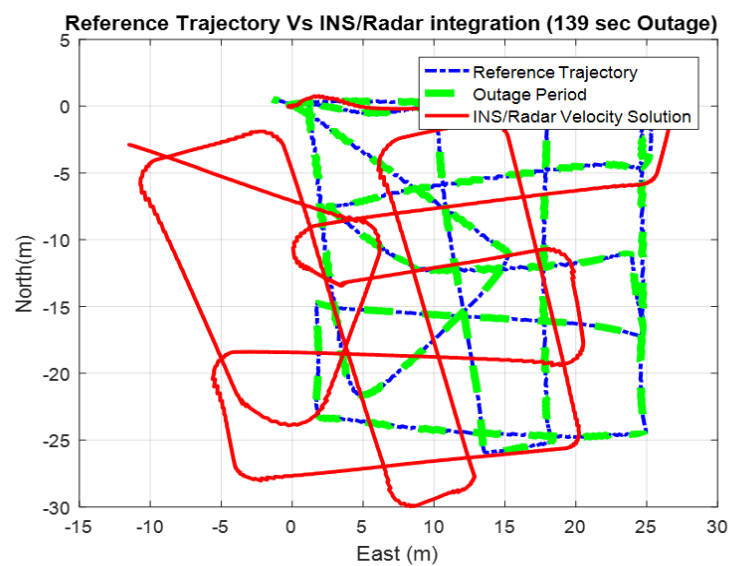

Figure 5. INS/Radar Odometry integration

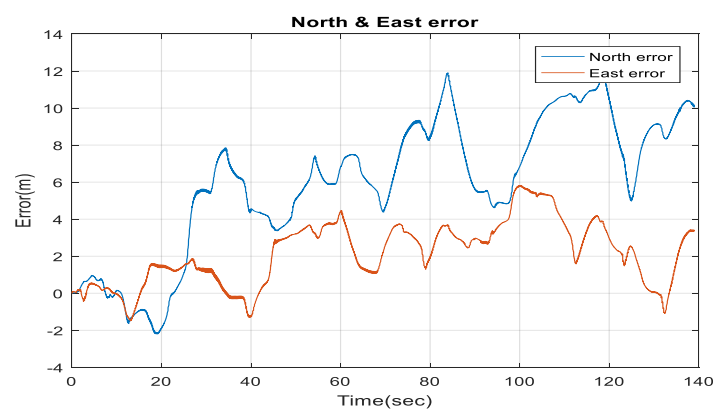

Figure 6. North and East error for INS/Radar odometry integration

\begin{tabular}{|c|c|c|c|}
\hline $\begin{array}{c}\text { RMSE-North } \\
(\mathrm{m})\end{array}$ & $\begin{array}{c}\text { RMSE-East } \\
(\mathrm{m})\end{array}$ & $\begin{array}{c}\text { Max-Error- } \\
\text { North }(\mathrm{m})\end{array}$ & $\begin{array}{c}\text { Max-Error- } \\
\text { East }(\mathrm{m})\end{array}$ \\
\hline 6.98 & 2.74 & 11.92 & 5.81 \\
\hline
\end{tabular}

Table 2. INS/Radar odometry integration performance

\subsection{Third test scenario}

This test considers (a) only the use of the UWB system, and (b) the combined use of the UWB system with radar to aid the INS. The purpose of this test is to check if the radar helps to enhance the navigation solution, when a large number of UWB anchors is available.

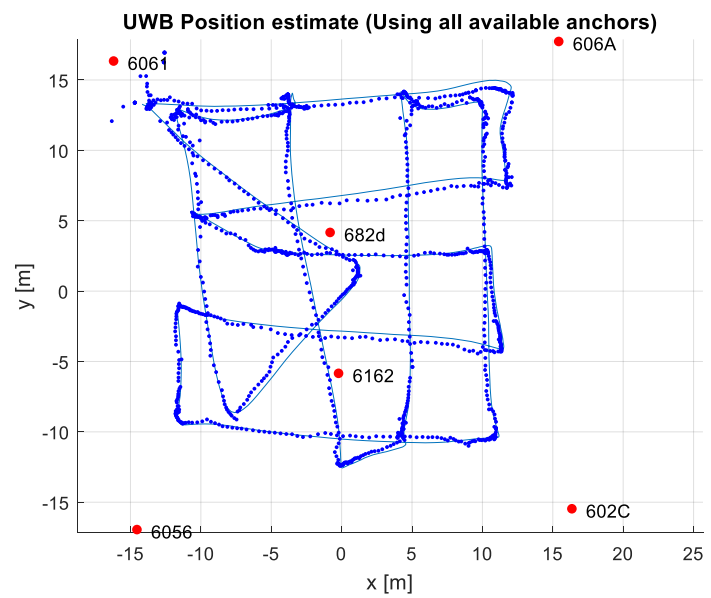

Figure 7. UWB position estimate, when using all available anchors in view

Figure 8 shows the UWB position estimate when using all the available UWB anchors. The maximum error reached, comparing the UWB position to GPS was $1.5 \mathrm{~m}$, but to reach this performance 6 UWB anchors were distributed in 30x30 meters area.

Figures 9 to 12 and table 3 show that in this experiment, where we have a large number of UWB anchors distributed all over the flight area, the radar does not enhance the navigation performance of the UAV. However, these results are obtained with a high density of UWB anchors: such kind of UWB infrastructure might be expensive and/or impracticable in a large area.

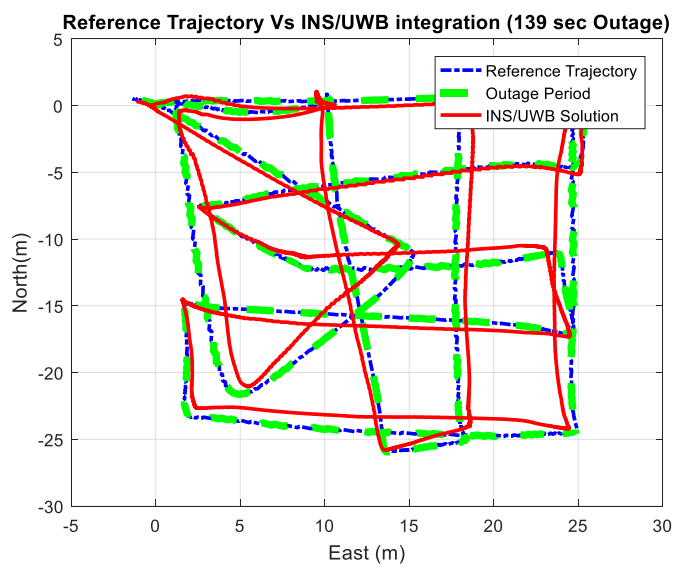

Figure 8. UWB integrated with INS solution (when all UWB anchors available where used)

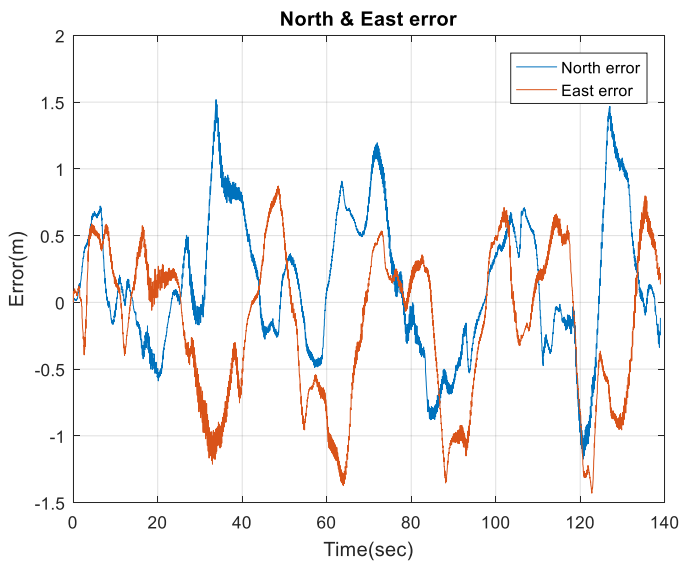

Figure 9. North and east error for UWB integration with INS (when all UWB anchors available were used)

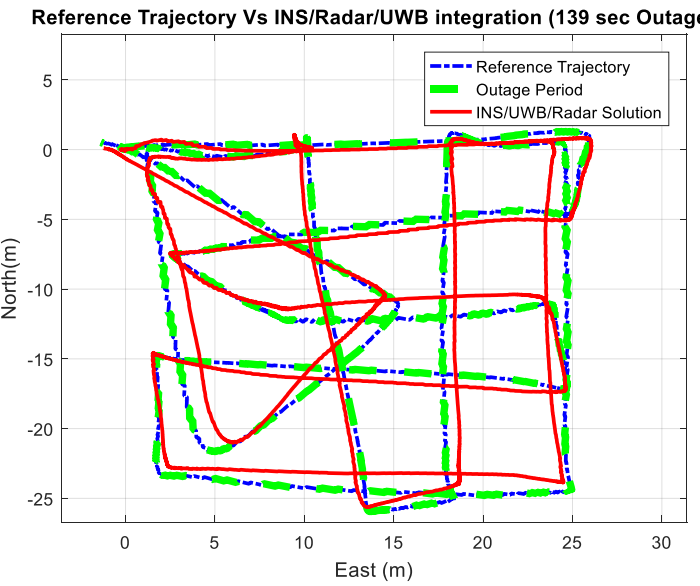

Figure 10. UWB/radar/INS integration (when all UWB anchors available were used) 


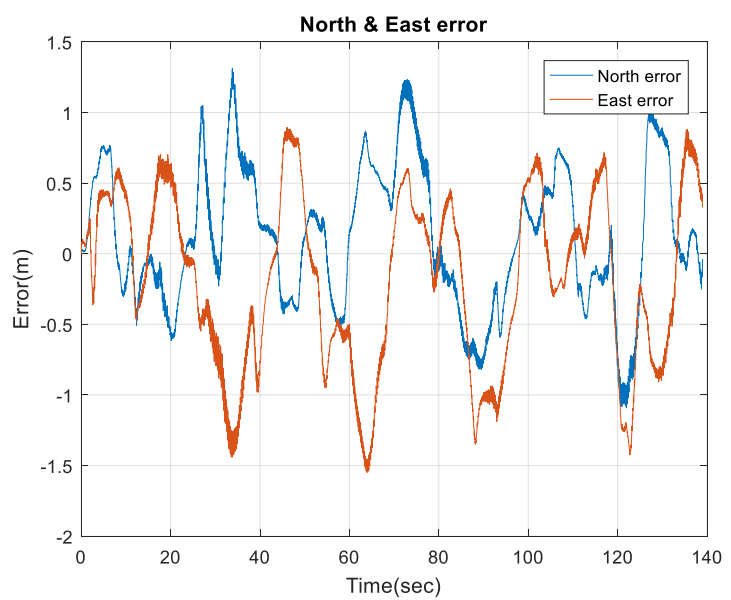

Figure 11. North and East error for UWB/radar/INS integration (when all UWB anchors available were used)

\begin{tabular}{|c|c|c|c|c|}
\hline & $\begin{array}{c}\text { RMSE } \\
\text { North }\end{array}$ & $\begin{array}{c}\text { RMSE } \\
\text { East }\end{array}$ & $\begin{array}{c}\text { Max-Error } \\
\text { North }\end{array}$ & $\begin{array}{c}\text { Max-Error } \\
\text { East }\end{array}$ \\
\hline UWB & $0.54 \mathrm{~m}$ & $0.59 \mathrm{~m}$ & $1.5 \mathrm{~m}$ & $0.72 \mathrm{~m}$ \\
\hline UWB/RO & $0.50 \mathrm{~m}$ & $0.63 \mathrm{~m}$ & $1.35 \mathrm{~m}$ & $1.51 \mathrm{~m}$ \\
\hline
\end{tabular}

Table 3. UWB/INS and UWB/radar/INS integration performance (when all UWB anchors available were used)

\subsection{Fourth test Scenario}

In this test scenario, measurements from only 4 UWB anchors were used in order to analyze the case of a lower UWB anchor density with respect to the previous case. The goal of this scenario is that of showing the benefits of the integration between UWB and radar to enhance the navigation performance in these working conditions, and hence enabling navigation in GNSS denied environments at lower infrastructure cost with respect to the previous scenario.

Figure 14 shows that in this case, the UWB position updates are not available along the entire UAV trajectory: since there are many time instants where UWB position estimates are not available, the estimated UAV trajectory frequently relies on the INS dead reckoning solution only, with a consequent significant reduction of the navigation performance with respect to the previous scenario.

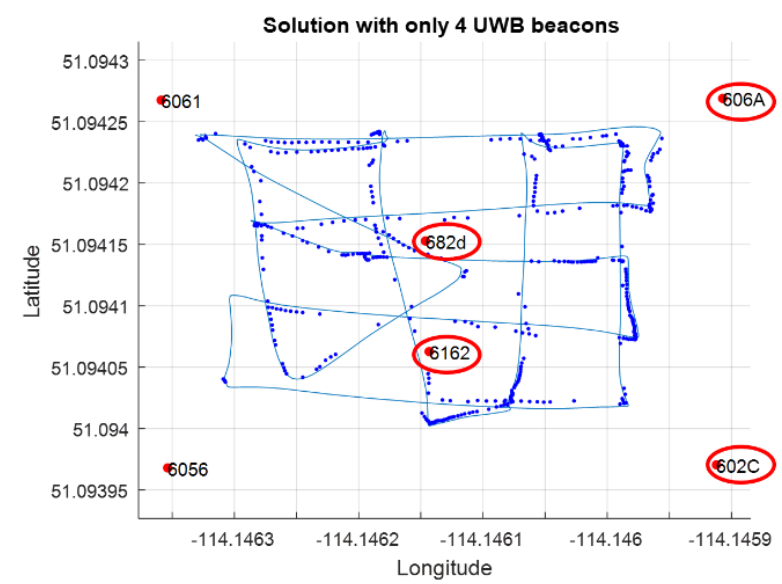

Figure 12. UWB position estimate when using 4 anchors only (position is accepted when 3 ranges are available)

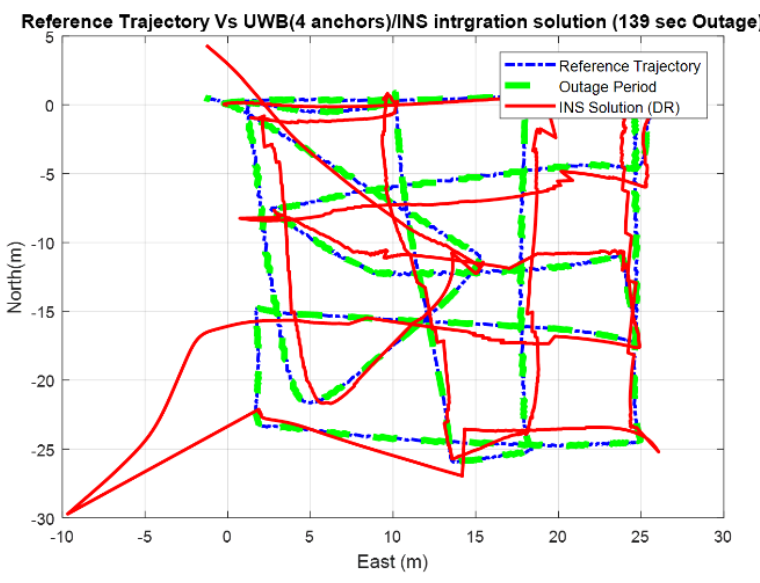

Figure 13. UWB integrated with INS solution (when four UWB anchors where used)

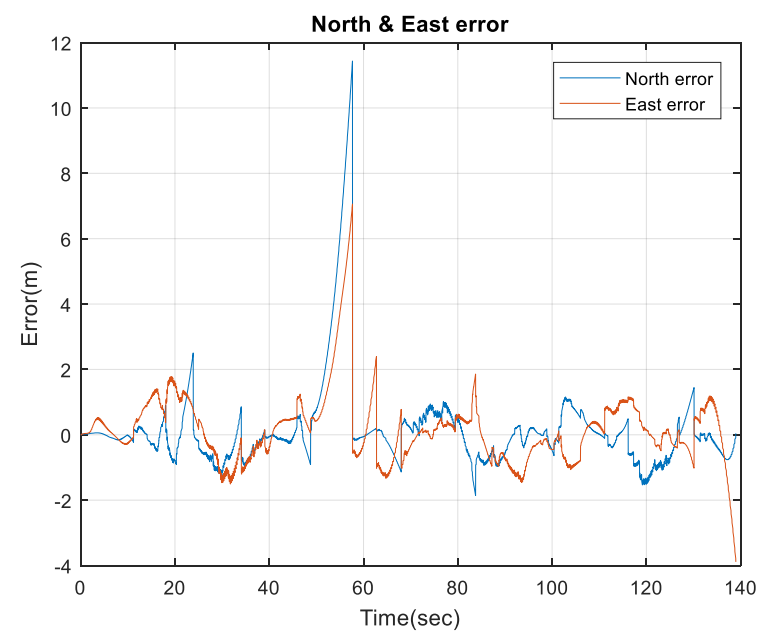

Figure 14. North and East error for UWB integrated with INS solution (when four UWB anchors where used)

The comparison between Figures. 14 and 16 and the results of Table 4 show that the integration between UWB and radar can be used to effectively enhance the navigation performance when the UWB infrastructure lacks the high density of UWB anchors previously considered in Scenario 3.

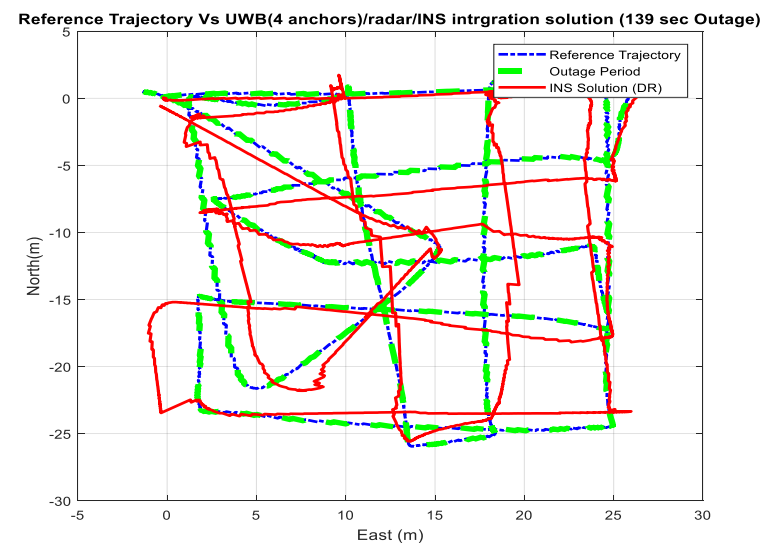

Figure 15. UWB/radar/INS integration solution (when four UWB anchors where used) 


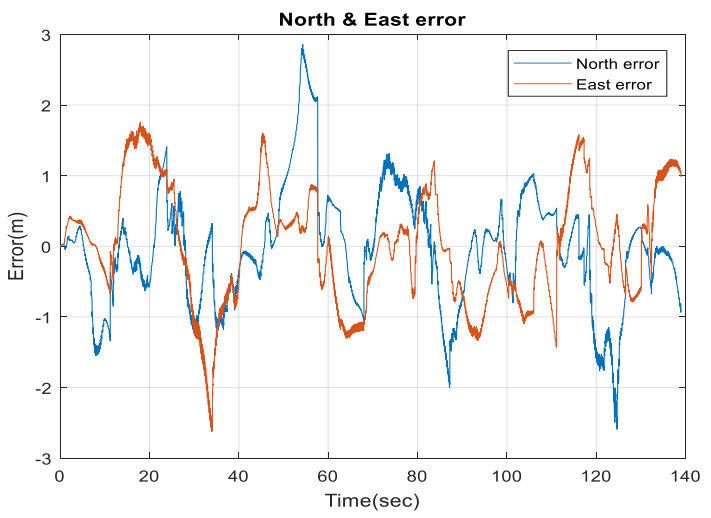

Figure 16. North and East error for UWB/radar/INS integration solution (when four UWB anchors where used)

\begin{tabular}{|c|c|c|c|c|}
\hline & $\begin{array}{c}\text { RMSE } \\
\text { North }\end{array}$ & $\begin{array}{c}\text { RMSE } \\
\text { East }\end{array}$ & $\begin{array}{c}\text { Max-Error } \\
\text { North }\end{array}$ & $\begin{array}{c}\text { Max-Error } \\
\text { East }\end{array}$ \\
\hline UWB & $1.48 \mathrm{~m}$ & $1.11 \mathrm{~m}$ & $11.43 \mathrm{~m}$ & $7.05 \mathrm{~m}$ \\
\hline UWB/RO & $0.83 \mathrm{~m}$ & $0.82 \mathrm{~m}$ & $2.85 \mathrm{~m}$ & $1.75 \mathrm{~m}$ \\
\hline
\end{tabular}

Table 4. UWB/INS and UWB/radar/INS integration performance (when four UWB anchors were used)

\subsection{Fifth test Scenario}

This test aims at (i) checking the effect of the spatial configuration of UWB anchors on the obtained navigation performance, (ii) confirming the results of the previous scenario but with a different UWB network configuration.

This test experimentally proves the effectiveness of the integration between radar and UWB systems to limit the error exhibited by the IMU, and hence reach better navigation performance when the number of available UWB anchors is lower than that in Scenario 3.

\begin{tabular}{|c|c|c|c|c|}
\hline & $\begin{array}{c}\text { RMSE } \\
\text { North }\end{array}$ & $\begin{array}{c}\text { RMSE } \\
\text { East }\end{array}$ & $\begin{array}{c}\text { Max-Error } \\
\text { North }\end{array}$ & $\begin{array}{c}\text { Max-Error } \\
\text { East }\end{array}$ \\
\hline UWB & $0.81 \mathrm{~m}$ & $1.02 \mathrm{~m}$ & $4.58 \mathrm{~m}$ & $6.59 \mathrm{~m}$ \\
\hline UWB/RO & $0.60 \mathrm{~m}$ & $0.64 \mathrm{~m}$ & $2.76 \mathrm{~m}$ & $1.35 \mathrm{~m}$ \\
\hline
\end{tabular}

Table 5.UWB/INS and UWB/radar/INS integration performance (when four UWB anchors were used)

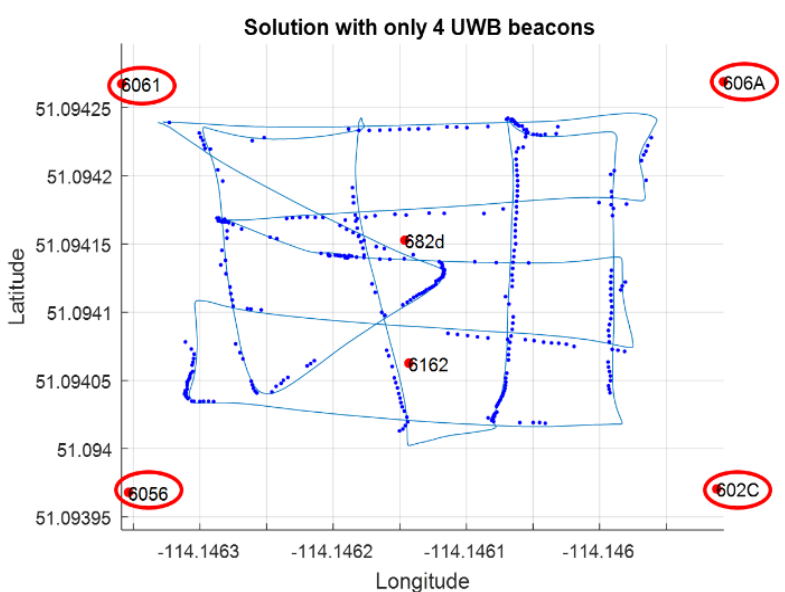

Figure 17.UWB position estimate when using 4 anchors only (position update is used only when at least 3 ranges are available)

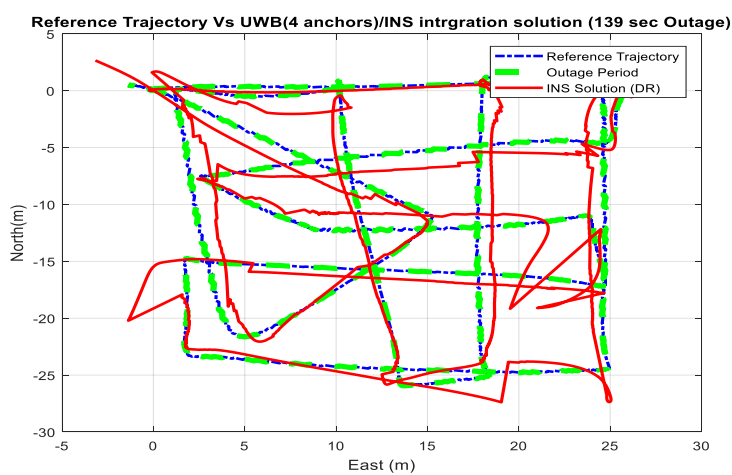

Figure 18. UWB integrated with INS solution (when four UWB anchors where used)

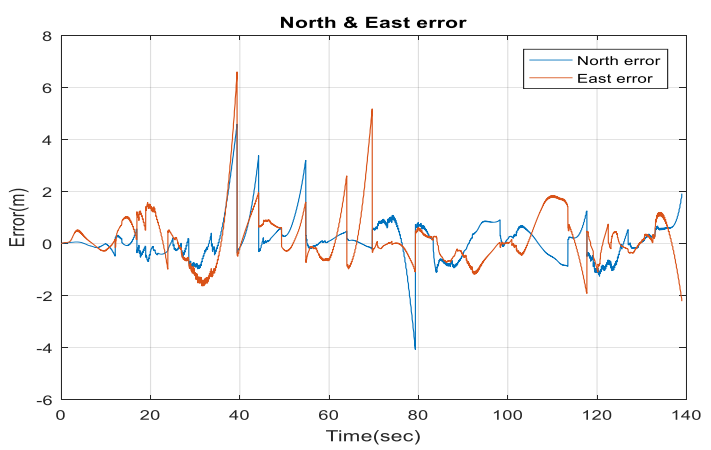

Figure 19. North and East error for UWB integrated with INS solution (when four UWB anchors where used)

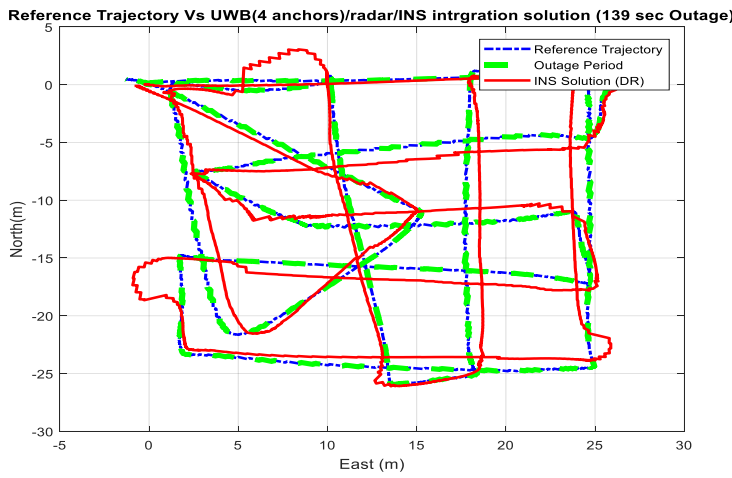

Figure 20. UWB/radar/INS integration solution (when four UWB anchors where used)

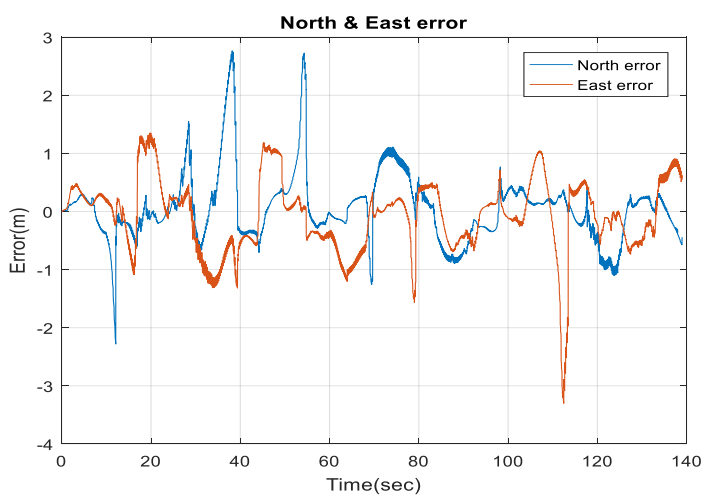

Figure 21. North and East error for UWB/radar/INS integration solution (when four UWB anchors where used) 


\section{CONCLUSIONS}

This paper presents the results obtained by the integration of a UWB and a micro radar system with INS, to aid the navigation of small UAV in GNSS denied environments. UWB and radar systems complement each other: usage of radar only does not provide sufficient information about the current heading direction, whereas the use of a standalone UWB system may require a huge infrastructure to yield acceptable position estimates during the entire flight. Experiments were performed by using an on-shelf commercial quadcopter ( $3 \mathrm{dr}$ Solo), with a micro FMCW radar tilted by 60 degrees and a UWB rover attached to its belly. Six UWB anchors were distributed on the ground, and their positions were surveyed with Trimble R10 GNSS receiver (accuracy $8 \mathrm{~mm} \mathrm{H} / 15 \mathrm{~mm} \mathrm{~V}$ ), hence enabling $3 \mathrm{D}$ rover position estimates by means of UWB measurements when at least four of them are available. The data from the radar (range doppler map) is binarized based on an experimentally set threshold, for targets detection and clutter removal purposes. The radial velocities for these detected targets are then obtained directly from the horizontal axis of the range-Doppler map, then the vehicle forward velocity is obtained after projecting estimated target velocities by the radar tilting angle, then taking the average of all the velocities.

Velocity from the radar, and positions from the UWB systems were integrated with INS solution, through EKF. Five test scenarios were presented in the result section to show the effectiveness of this integration when the density of UWB anchors is not high.

In scenario 4 (results obtained by using four UWB anchors), the integration between radar and UWB showed $44 \%$ and $26 \%$ enhancement in the RMSE-North and RMSE-East direction respectively, when compared to the UWB solution only. Similarly, in scenario 5 (with four UWB anchors but distributed differently with respect to scenario 4) the RMSE-North and RMSE-East were enhanced by $25 \%$ and $37 \%$ respectively, hence experimentally proving the convenience of the proposed solution also for different configurations of the UWB anchor network.

\section{ACKNOWLEDGMENT}

This work was supported by Dr. Naser El-Sheimy research funds from NSERC and Canada Research Chairs programs.

\section{REFERENCES}

Aqel, M. O. A., Marhaban, M. H., Saripan, M. I., \& Ismail, N. B. 2016. Review of visual odometry: types, approaches, challenges, and applications. SpringerPlus.

Barrett, D., \& Alvarez, A. 2017. mmWave radar sensors in robotics applications.

Cadena, C., Carlone, L., Carrillo, H., Latif, Y., Scaramuzza, D., Neira, J., Leonard, J. J. 2016. Past, Present, and Future of Simultaneous Localization and Mapping: Toward the RobustPerception Age. IEEE Transactions on Robotics, 32(6), 13091332.

Fasano, G., Renga, A., Vetrella, A. R., Ludeno, G., Catapano, I., \& Soldovieri, F. 2017. Proof of concept of micro-UAV-based radar imaging. In 2017 International Conference on Unmanned Aircraft Systems (ICUAS) (pp. 1316-1323).

Gao, Y., Liu, S., Atia, M. M., \& Noureldin, A. 2015. INS/GPS/LiDAR Integrated Navigation System for Urban and Indoor Environments Using Hybrid Scan Matching Algorithm. Sensors (Basel, Switzerland), 15(9), 23286-23302.
Goel, S., Kealy, A., Retscher, G., \& Lohani, B. 2016. Cooperative P2I localization using UWB and Wi-Fi. In Proceedings of International Global Navigation Satellite Systems (IGNSS) Association Conference (pp. 6-8).

Gross, J. N., Gu, Y., \& Rhudy, M. B. 2015. Robust UAV relative navigation with DGPS, INS, and peer-to-peer radio ranging. IEEE Transactions on Automation Science and Engineering, 12(3), 935-944.

Kauffman, K., Raquet, J., Morton, Y., \& Garmatyuk, D. 2013. Real-Time UWB-OFDM Radar-Based Navigation in Unknown Terrain. IEEE Transactions on Aerospace and Electronic Systems, 49(3), 1453-1466.

Kim, E., \& Choi, D. 2016. A UWB positioning network enabling unmanned aircraft systems auto land. Aerospace Science and Technology, 58, 418-426.

Kumar, G. A., Patil, A. K., Patil, R., Park, S. S., \& Chai, Y. H. 2017. A LiDAR and IMU Integrated Indoor Navigation System for UAVs and Its Application in Real-Time Pipeline Classification. Sensors (Basel, Switzerland).

Li, H., Zhong, Z., Kong, W., \& Zhang, D. 2015. A fast calibration method for autonomous landing of UAV with ground-based multisensory fusion system. In Information and Automation, 2015 IEEE International Conference on (pp. 3068-3072). IEEE.

Mohamed, H. A., Moussa, A. M., Elhabiby, M. M., El-Sheimy, N., \& Sesay, A. B. 2016. IMPROVED REAL-TIME SCAN MATCHING USING CORNER FEATURES. ISPRS International Archives of the Photogrammetry, Remote Sensing and Spatial Information Sciences, XLI-B5, 533-539.

Masiero, A., Fissore, F., Vettore. A., 2017. A low cost UWB based solution for direct georeferencing UAV photogrammetry. Remote Sensing, 9(5), 414.

Mostafa, M., Moussa, A., El-Sheimy, N., \& Sesay, A. B. 2017. Optical Flow Based Approach for Vision Aided Inertial Navigation Using Regression Trees, Retrieved December 14.

Nistér, D., Naroditsky, O., \& Bergen, J. 2004. Visual odometry. In Computer Vision and Pattern Recognition. CVPR 2004. Proceedings of the 2004 IEEE Computer Society Conference on (Vol. 1, pp. I-I). Ieee.

Pozyx Labs, Pozyx Positioning System. Available online: https://www.pozyx.io/

Scannapieco, A. F., Renga, A., Fasano, G., \& Moccia, A. 2016. Ultralight radar sensor for autonomous operations by microUAS. In 2016 International Conference on Unmanned Aircraft Systems (ICUAS) (pp. 727-735).

Schauwecker, K., \& Zell, A. 2014. On-Board Dual-Stereo-Vision for the Navigation of an Autonomous MAV. Journal of Intelligent \& Robotic Systems, 74(1-2), 1-16.

Shin, Y. H., Lee, S., \& Seo, J. 2017. Autonomous safe landingarea determination for rotorcraft UAVs using multiple IR-UWB radars. Aerospace Science and Technology, 69, 617-624.

Tiemann, J., Schweikowski, F., \& Wietfeld, C. 2015. Design of an UWB indoor-positioning system for UAV navigation in GNSS-denied environments. In Indoor Positioning and Indoor Navigation (IPIN), 2015 International Conference on (pp. 1-7). IEEE. 